\title{
Une fable humaniste : la conquête de l'île de nulle part, de More à Shakespeare
}

\section{Raymond Gardette}

\section{(2) OpenEdition}

\section{Journals}

Édition électronique

URL : http://journals.openedition.org/shakespeare/1269

DOI : 10.4000/shakespeare.1269

ISSN : 2271-6424

Éditeur

Société Française Shakespeare

Édition imprimée

Date de publication : 1 novembre 1994

Pagination : 15-33

Référence électronique

Raymond Gardette, «Une fable humaniste : la conquête de l'île de nulle part, de More à Shakespeare », Actes des congrès de la Société française Shakespeare [En ligne], 12 | 1994, mis en ligne le 01 janvier 2007, consulté le 01 mai 2019. URL : http://journals.openedition.org/shakespeare/1269 ; DOI : 10.4000/shakespeare.1269 
COSMOPOLITISME ET INSULARITÉ 


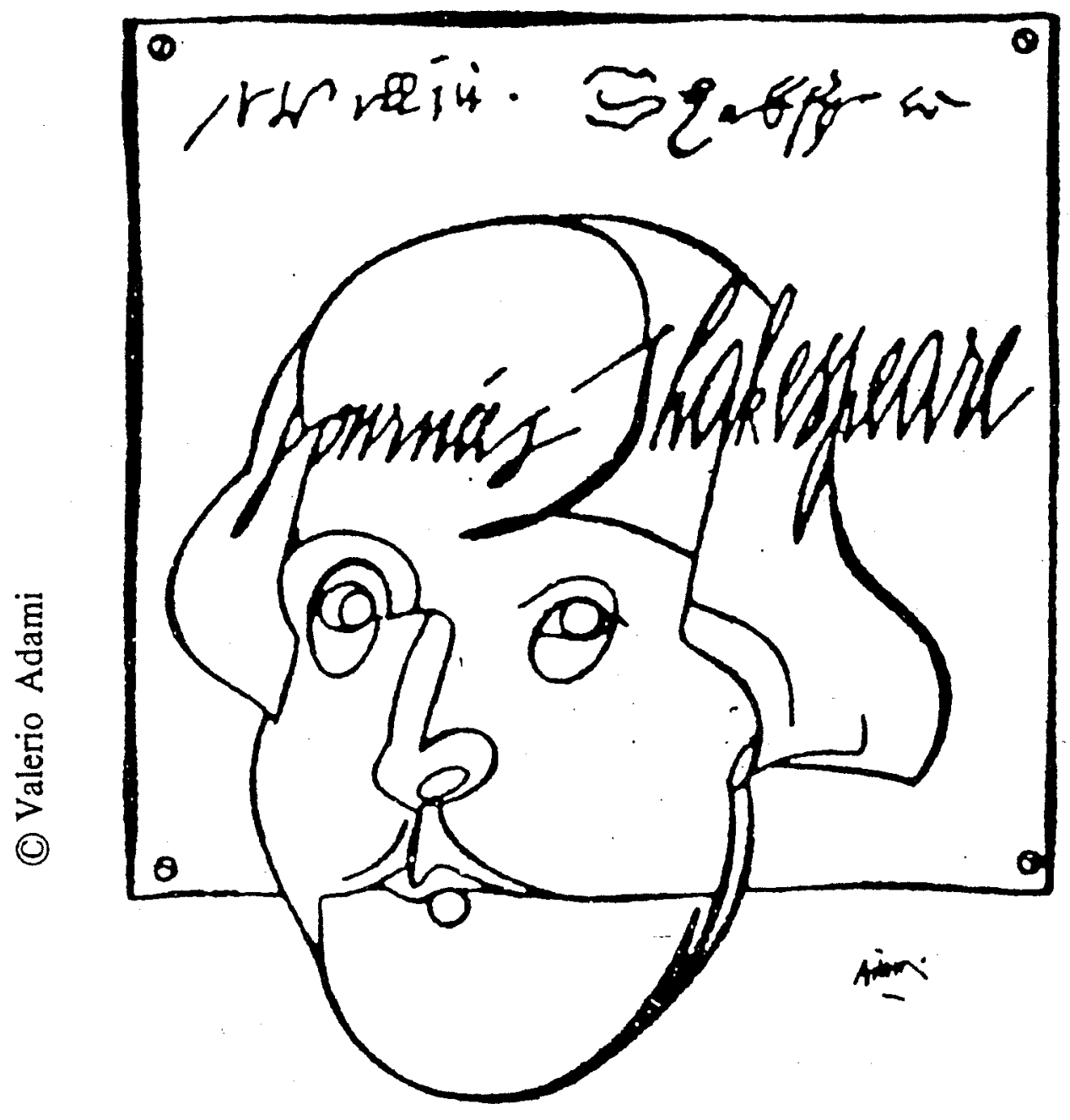

Affiche de Valerio Adami, spécialement conçue pour les journées Shakespeare 1979 


\section{SHAKESPEARE}

COSMOPOLITISME ET INSULARITÉ

Société Française Shakespeare

Actes du Congrès 1993

sous la direction

de

M. T. JONES-DAVIES

Ouvrage publié avec le soutien de

BARCLAYS

BARCLAYS BANK S.A

PARIS

LES BELLES LETTRES

1994 
Tous droits de traduction, de reproduction et d'adaptation réservés pour tous les pays.

(C) 1994 Société d'édition Les Belles Lettres, 95 bd Raspail 75006 Paris

ISBN 2.251.69122-7 


\title{
Une fable humaniste : la conquête de l'île de nulle part, de More à Shakespeare.
}

\author{
And prompt me plain and holy innocence (Miranda) \\ And my ending is despair \\ Unless I be relieved by prayer (Prospero)
}

Dans la tradition héritée des récits de voyage du moyen âge, le voyageur du seizième siècle a conscience d'aller à la découverte des merveilles et prodiges de la Création, où le monstrueux côtoie le prodigieux, le reconnaissable l'invraisemblable et le naturel le surnaturel. Ce qu'a de spirituel l'attitude du navigateur est extériorisé dans la description géographique ${ }^{1}$. Très souvent utilisée, la référence à Ophir, symbole des richesses éternelles pour lesquelles les découvreurs de terres nouvelles endurent tempêtes et naufrages ${ }^{2}$, indique que la démarche du navigateur est, en tout premier lieu, d'ordre divin. A l'orée du dix-septième siècle, Samuel Purchas, dans l'immense compilation dont il adresse la troisième édition à Jacques $1^{\mathrm{er}}$, inscrit la longue série de voyages antiques et modernes sous une référence qu'il dit être "allégorique et anagogique", à la navigation de Salomon :

Every Christian man is a ship, a weak vessel, in this navie of Solomon and dwelling in a mortal body, is within lesse then four inches, then one inch of death ${ }^{3}$.

Les axiologies contrastées qu'offre La Tempête, où le cadre méditerranéen prend l'allure d'une île de l'Atlantique, permettent d'associer l'affabulation utopique et un discours allégorique sur les limites humaines. En premier lieu, le récit, ou organisation du matériau littéraire, suit la convention du genre pastoral, où les frontières spatiales et temporelles ont licence de s'effacer. Ainsi, Ariel à la façon des autres apparitions surnaturelles dans les dernières comédies de Shakespeare ou encore des figures divinisées qui côtoient les courtisans dans les Masques de la cour jacobéenne, évoque-t-il la rupture magique des bornes humaines du proche et du 
lointain, du visible et de l'invisible. Lorsque se termine l'acte magique, il fait réapparaître le navire royal et son équipage :

Before you can say 'come' and 'go',
And breathe twice, and cry 'so, so',
Each one, tripping on his toe,
Will be here with mop and mow.

Ariel symbolise le désir de découverte par l'effacement des limites temporelles et spatiales, l'ouverture utopique vers l'Ailleurs, en complicité avec Prospero:

I drink the air before me, and return

Or ere your pulse twice beat

Cet agencement de surface renvoie à une structure profonde, un discours d'ordre humaniste, dont le thème central est le pèlerinage imaginaire, à partir de la fiction de l'île non encore répertoriée par les navigateurs et les cartographes. Un monde non encore existant est, pour ainsi dire, la création du découvreur malgré lui. Le but ultime de cette fable dramatique et géographique est une mise à distance de la sphère politique, un avertissement en termes de théâtre, face au danger spirituel que recouvre l'usurpation sous toutes ses formes. Dans la mesure où Prospero, par son art, réussit à dominer les hommes et à vaincre les esprits, il sacralise l'intégrité politique.

L'île de La Tempête, comme l'Utopie de Thomas Mores, à quelque cent ans de distance, propose au souverain de l'époque la vision en réfraction de ce que devient un royaume lorsque l'équité ne tempère pas la justice ${ }^{6}$. De même que l'utopie morienne oppose la vision d'une île imaginaire (livre II), à la description de l'Angleterre d'Henri VIII (livre I), de même la représentation théâtrale permet-elle, grâce aux possibilités dramatiques inhérentes au choix du lieu, celui d'une île inconnue, de montrer le contraste entre l'innocence et le mal et de dévoiler le mécanisme des divers asservissements de l'esprit qui se déclenche, dès lors que l'illégitime le dispute au légitime. L'aventure de Prospero est une leçon d'humanisme. A ce titre, elle comporte, nécessairement, une part d'insatisfaction de sorte qu'une certaine part d'ambiguïté se mêle à l'ironie jusqu'au terme du spectacle? 
Une première convention de l'écriture utopique est le postulat du récit authentifié par des témoins objectifs, grâce auquel le relais de l'invention s'inscrit dans un cadre spatial et/ou temporel reconnaissable. La Tempête établit, avec insistance, des repères dans la vie passée de Prospero. Ce dernier décrit son sauvetage miraculeux face aux éléments déchaînés, par "Providence divine" (I; ii. 159), grâce à une reconstitution du passé, où l'épisode antérieur est présenté comme une scène revécue. Cet appel à l'anamnèse permet de reconstituer ce tiers de la vie de Prospero qui fut le cadre de l'enfance et de l'éducation de Miranda ${ }^{8}$. La nécessité de revivre son passé par la mémoire, donc dans le cas de Miranda, de lui faire découvrir le temps où elle était fille de duc, donne à l'histoire de Prospero son véritable caractère de fable politique. Le maître de l'île a soin de reprendre le récit interrompu de son exil et il ne cesse de rappeler à Ariel et à Caliban les raisons et les conditions de leur obéissance. Ces serviteurs involontaires jouent, au cours du voyage imaginaire, le rôle de ce que T. S. Eliot appelait "objective correlative". Ils apportent une caution de vérité au sein de l'utopie.

Le prétexte utopique prend un appui spatial chez Thomas More, où la prétendue circumnavigation d'Hythlodée n'est qu'une description prospective du tour du monde d'ouest en est, réussi de 1519 à 1522 par Jean Sébastien de El Cano, lieutenant de Magellan - lequel mourut au cours du voyage, sort que partagent deux des compagnons d'Hythlodée (Ut., 662). Celui-ci aurait passé cinq années sur l'île d'Utopie, à la façon de John Rastell, dramaturge et cosmographe, beau-frère de Thomas More, vers $1517^{\circ}$. L'Utopie emprunte aux Quatuor Navigationes d'Amerigo Vespucci, le compagnon prétendu d'Hythlodée, des détails de navigation et de découverte. L'habileté consiste à faire croire à l'existence de terres, jusque là inconnues, que les auteurs anciens n'auraient pu mentionner, comme le prouve, par exemple, chez Dante, la référence à une mer océane sans habitants ${ }^{10}$. Grâce à la présence de témoins dignes de foi, More renforce la supercherie. Il demande des précisions sur les dimensions du pont d'Amaurote au-dessus du fleuve Anydre (Ut., 349), et Pierre Gilles donne une caution d'objectivité à l'affabulation géographique :

Quant à l'objection que le nom de cette île ne se trouve nulle part chez les auteurs cosmographes, Hythlodée y a très bien répondu lui-même : il est fort possible, dit-il, que le nom qu'utilisaient les anciens ait été changé par la suite; ou encore que cette 
île ait échappé à leur attention, de la même façon qu'aujourd'hui se révélent un bon nombre de terres nouvelles inconnues des anciens géographes.

(Ut., 341)

Une cartographie en train de se faire, tel est le présupposé de la description utopique. Les difficultés que pose à la conscience chrétienne la découverte du nouveau monde se relient à cette convention du monde à découvrir. Le voyage d'Hythlodée retient des éléments se rapportant à la colonisation des Amériques, comme en témoigne la révision, en 1517, après la publication de l'Utopie, des projets présentés deux années auparavant par Bartholomé de Las Casas à la cour d'Espagne ${ }^{11}$. Le nouveau monde sert de prétexte à la dénonciation des iniquités en terre chrétienne. Il semble que La Tempête soit dans la droite ligne de cette tradition.

Un deuxième postulat caractérise l'écriture utopique : la connivence entre l'auteur et son lecteur. L'attitude humaniste consiste à feindre de mettre l'affabulation au service de la vérité, de façon à ce que le lecteur en ait conscience. Dans sa lettre à Pierre Gilles, More distingue entre le prétexte du mensonge et le mensonge réel :

[...] autant je mettrais tous mes soins à éviter que le livre ne contienne quelque erreur, autant, si j'hésitais sur quelque point, j'aimerais mieux dire un mensonge que commettre un mensonge, préférant manquer à la sagacité plutôt qu'à l'honnêteté. (Ut., 20)

"Dire un mensonge" [mendacium dicere], appartient au style du récit et au genre littéraire utilisé, mais "commettre un mensonge" [mentiri], parce qu'on trompe le lecteur, enfreint l'ordre moral et spirituel. La Tempête, comme L'Utopie, fait usage de ce sophisme. L'affabulation d'une aventure sur une île inconnue, où l'irréalisable prend une allure objective, se présente comme le résultat d'une complicité entre le dramaturge et son auditoire. Sur la scène shakespearienne, des illusions se créent et se défont, sous l'égide de la licence théâtrale. Ainsi s'établit une méta-utopie, au déroulement de laquelle veille l'esprit Ariel. Ce dernier dans le langage de son maître et sous ses ordres, convient de la nécessité de "dire" des mensonges afin d'éprouver la résistance spirituelle de Ferdinand et aussi de mettre à jour les instincts meurtriers des usurpateurs, courtisans ou grotesques, mais il admet l'interdiction de "commettre" un mensonge. La théurgie, grâce à laquelle Prospero établit des liens avec les forces cachées de l'univers, ne souffre aucun 
manquement à la loyauté chez les serviteurs du magicien. Ariel en est conscient, puisqu'il prend soin de rappeler à son maitre l'étendue de sa loyauté : «I prithee, / Remember I have done thee worthy service, / Told thee no lies, made no mistakings, ...» (I. ii. 246-8). Prospero, en imposant à son serviteur un devoir de fidélité, installe l'illusion dramatique dans la catégorie du véridique. Sur scène, les spectacles illusoires qu'organise le magicien dans le cercle du théâtre sont de l'étoffe même dont est faite l'utopie.

La distance entre ficiton et message, autre caractéristique essentielle de l'utopie, est ce qui permet le décèlement des insuffisances de la cité terrestre. La méthode utopique repose sur la confrontation paradoxale des contraires. C'est de l'opposition du monde irréel au monde de l'action que naît le désir d'utopie (Ut., 179-84). L'évocation d'une île non-existante, siège d'une société idéale où cesse l'injustice, où la guerre est une juste cause, où, selon More, l'esclavage demeure dans les limites du droit, fait apparaître, par réflection et contraste, les exactions, les iniquités, les injustices du royaume existant. Le livre I de l'Utopie est, à ce titre, un martyrologe du peuple anglais, présenté sous la forme d'une dystopie, c'est-à-dire de la description de l'injustice, de la misère, de la cupidité, de la violence, de la guerre telles qu'elles sont. Ce livre I se lit à la lumière du livre II, lequel est la description d'une société fictive, idéale et irréalisable. L'île, dans La Tempête, joue ce même rôle de cadre matriciel, où le retour des coupables prend son sens dans leur confrontation avec l'utopie créée par Prospero. L'irréel, sous la forme d'une île illusoire, d'une tempête fictive, de Masques joués par des esprits, est destiné à dévoiler et dénoncer les mécanismes de l'usurpation. Le thème, dans les deux œuvres, est celui du theatrum mundi, la scène-spectacle du monde. Selon More, le philosophe a un rôle à tenir, à condition qu'il sache composer avec les contigences politiques, tout en évitant le dogmatisme. La philosophie scolastique manque de souplesse; il en existe une autre, nous dit l'humaniste, qui doit savoir s'adapter à la scène du monde, car elle est

... mieux instruite de la vie en société : elle connaît son théâtre et elle s'y accommode ; dans la pièce, elle accepte le rôle qui lui revient et le joue avec beaucoup d'élégance et de grâce. (Ut., 430-33)

Dans la pièce de Shakespeare, Prospero est une figuration dramatique de ce philosophe politique. Le naufrage qu'il met en scène 
s'inspire de la méthode prônée par Thomas More, selon laquelle le philosophe se doit, même s'il connaît des échecs, de ne jamais relâcher son combat contre les «abus consacrés par l'usage ... pas plus qu'on ne doit abandonner un navire en pleine tempête» (Ut., 433). Prospero, en contraignant les voyageurs à quitter le navire royal, dénonce leur illégitimité, puisqu'il les condamne à subir l'insolence des éléments déchaînés. Le maître d'équipage a beau jeu d'avertir ses nobles passagers de l'indifférence des rugissants quant aux hiérarchies humaines: "What care these roarers for the name of King ?» (I. i. 16-17). La fiction du naufrage a valeur de message politique.

L'ironie est le mode d'écriture privilégié de la fable utopique. Le face à face de deux mondes antithétiques, l'un irréel, illusoire, imaginaire, l'autre réel, reconnaissable, précis, fonctionne comme un palimpseste qui aurait valeur de réquisitoire. Le monde imaginaire, cadre d'un spectacle, métaphorise le monde réel durant le temps de la représentation. Le propos utopique entend, par ses détours, dévoiler aux yeux du lecteur/spectateur les conditions d'existence des erreurs, des iniquités, des crimes politiques. A l'intérieur de la construction utopique, la découverte de sociétés fictives, le spectacle d'illusions magiques ont valeur de maïeutique. Il est question, par l'entremise de l'utopie, de faire naître un monde à lui-même. De façon significative, La Tempête organise un spectacle illusoire, à l'intérieur de l'illusion du spectacle, afin de mettre un terme indispensable à la vision idéalisée de la société humaine. La construction dramatique pose les limites de l'innocence sur la toile de fond de la corruption et de l'usurpation. Ainsi l'intrigue amoureuse entre Ferdinand et Miranda renvoie-t-elle aux contradictions initiales. L'union des deux jeunes gens renouvelle ironiquement la soupission de Milan à Naples. En outre, elle fournira l'occasion d'un spectacle magique qui se verra brutalement interrompu :

Let me live here ever;

So are a wonder'd father and a wife Makes this place paradise. (IV. i. 123-5) ${ }^{12}$

Le désir d'utopie formulé par Ferdinand ne peut que rester vain. Il ne sert, en fin de compte, qu'à mettre en relief l'irréfragable condamnation de Milan et de Naples. En secret, Prospero va créer le prétexte de diverses formes de l'usurpation, toutes irréalisables, et 
il en surveille l'évolution, réduisant ainsi la vie humaine à un simple Masque, à un spectacle que l'on peut inventer et contrôler. Les grotesques, qui rééditent sur un mode mineur les motivations usurpatrices - le désir, dans l'illégitimité, d'être roi, de gouverner des sujets, d'établir un empire - demeurent conscients, à la façon de "morosophes" (Ut., 38) des dangers qu'ils font courir à l'État :

Trin. Servant-monster! The folly of this island! They say there's but five upon this isle: we are three of them; if th'other two be brain'd like us, the state totters. (III. ii. 4-6)

L'accouchement monstrueux ${ }^{13}$, à partir de trois corps avinés et entremêlés, est un emblème graphique de la monstruosité politique. Tout concourt, sur l'île de Prospero, comme cela est le cas dans la confrontation des livres I et II de l'Utopie, à dénoncer les dangers d'une colonisation de l'esprit en dehors des voies de la justice tempérée par l'équité. Prospero, en philosophe humaniste conscient des turpitudes humaines, ne peut que mettre fin à l'affabulation utopique, abandonner les attraits de la magie, mettre fin au spectacle. «Our revels are now ended» (IV. i. 148) : l'aventure utopique est indissolublement liée à un voyage-retour et les limites du monde nouveau sont, en définitive, celles de l'ancien monde.

La cartographie de l'île utopique répond aux exigences de l'écriture ironique. L'île est un non-territoire, au nom inscrit à l'envers dans les premières cartes portulans ${ }^{14}$, par étymologie an-

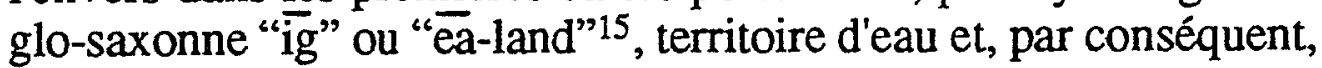
ouvert sur l'Ailleurs. Elle permet d'associer le fini et l'infini, le tangible et l'intangible, le réel et l'imaginaire. Dans la gravure de 1518 représentant lîle d'Utopie, reflet inversé du premier dessin de $1516^{16}$, le spectateur est face à un monde à l'envers, que regardent, au premier plan, le navigateur Hythlodée et un soldat prêt à la conquête, deux conquérants de linutile. Le troisième personnage, Thomas More, tourne le dos à l'île-reflet et dirige son regard vers l'infini. Le graphisme souligne l'importance d'un tel initiateur, aux visées intérieures $(U t ., 332)$. Prospero joue ce même rôle de philosophe capable de voir la cité utopique avec les yeux de l'esprit. Le reproche d'usurpation qu'il adresse à Ferdinand peut se lire comme la récusation de toute idée de conquête de terres nouvelles qui ne serait pas motivée par une réflexion humaniste. L'île utopique n'a aucune réalité géographique. Elle n'est pas une terre à découvrir. 
Ou-topia, non-lieu, eu-topia, lieu du bonheur, ou oudetopia, lieu du jamais, les explications étymologiques savantes appliquées à l'île inventée par Thomas More (Ut., 673, p. 70) ne sont pas les signifiants d'un pays signifié. Utopia est non-Abraxa, une île créée par détachement du continent. Le seul signifié est la distance par rapport au monde connu ${ }^{17}$. L'île de Thomas More est située dans l'hémisphère austral, mais elle ne répond à aucun référent géographique précis ( $U t ., 366-9$ et 562). Pour Guillaume Budé, elle est Hagnopolis, nouvelle Jérusalem, «sans doute l'une des îles fortunées, proche peut-être des Champs Elysées» (Ut., 325). Dans la pièce de Shakespeare, l'indétermination géographique rend possible une mise en scène sous l'égide de la magie. La flotte royale se retrouve dispersée en Méditerranée alors que le navire royal, ("king's ship"), est immobilisé aux Bermudes avec son équipage, sous l'effet d'un charme (I. ii. 224-37). Le passage de l'équateur est allégoriquement évoqué par les grotesques, à partir d'un jeu scénique et verbal sur le mot "line", non seulement la corde qui soutient les habits royaux que se disputent Stephano et Gonzalo, (ou celle à laquelle Gonzalo avait promis le maître d'équipage) mais aussi la ligne équinoxiale et, plus encore, la limite infranchissable de la cellule de Prospero, l'imprescriptible frontière de la connaissance humaniste (IV. i. 235-45 ; V. i. 10). L'île shakespearienne est le cadre paradoxal de reflets inversés. Le monde nouveau croit découvrir chez les politiques corrompus de l'ancien monde un "brave new world". Ces derniers opposent leur scepticisme à la description de l'Age d'Or que fait Gonzalo (II. i. 122-66), après Montaigne ${ }^{18}$, alors que ces mêmes politiciens prennent les illusions magiques pour des réalités géographiques, signe inaliénable de la corruption de leur esprit. Le nouveau monde stigmatise ainsi les insuffisances de l'ancien. Montaigne, ayant rencontré à la cour de Charles IX à Rouen, en 1562, trois "sauvages" issus de "La France Antarctique" - la côte actuelle du Brésil - où avait abordé l'expédition conduite par Villegagnon, rapporte ironiquement l'étonnement de ces "cannibales" face à notre monde,

ignorans combien coutera un jour à leur repos et à leur bon heur la connaissance des corruptions de deça, et que de ce commerce naistra leur ruyne, comme je presuppose qu'elle soit desjà avancée, bien misérables de s'être laissez piper au désir de la nouvelleté, et avoir quitté la douceur de leur ciel pour venir voir le nostre. 
L'auteur des Essais garde en mémoire deux exemples de ce qu'il nomme des "choses admirables" décrites par ces Indiens - le fait qu'un peuple civilisé pût se laisser gouverner par un enfant et qu'un pays ne sût partager ses richesses entre riches et pauvres ${ }^{19}$.

La Tempête s'organise autour de telles confrontations. Dans son nom même, où l'on peut lire chalyb (eates), can(n)ibal, calib = carib (caribéen) ${ }^{20}$, Caliban associe échos mythiques et référents aborigènes. Sa vie est un mélange d'obéissance servile et de révolte justifiée, l'affirmation d'une cynique et grossière sensualité aux côtés d'une authentique sensibilité pastorale. En un sens, Caliban, comme les "cannibales" de Montaigne, est susceptible d'être corrompu par des colonisateurs, soucieux de monnayer son esclavage (II. ii. 28-34). La seule protection de "cet être de ténèbres" (V.i. 275) est celle que lui accorde Prospero, philosophe averti des vilenies politiques. Le duc de Milan, lorsqu'il recouvre sa légitimité face à un frère usurpateur (V. i. 130-34), revendique le "calibanesque" comme partie intégrante de l'humaine condition. C'est ainsi que l'île mystérieuse et magique, en raison même de son indétermination géographique, s'est faite le creuset d'une réflexion sur les enjeux du pouvoir en même temps que sur les limites de la conquête des territoires et des esprits.

La thématique de l'abandon de l'île utopique recouvre, semble-t-il, des échos de l'histoire mouvementée des tentatives colonisatrices infructueuses de l'Angleterre élisabéthaine. Lorsque Jacques $1^{\text {er }}$, lors des fetes d'hiver de 1611 et, un an et demi plus tard, à l'occasion des festivités organisées à l'occasion du mariage de la princesse Elisabeth avec l'électeur Palatin, accueille à la cour les fantasmagories de La Tempête, Walter Ralegh est en prison. L'auditoire royal de Shakespeare a en mémoire le projet colonisateur du demi-frère de Sir Humphrey Gilbert, d'abord accepté par Elisabeth, à condition qu'il s'agît de terres vierges de toute présence humaine. Wingandacoa fut appelée Virginie en l'honneur de la souveraine, et les premiers planteurs crurent avoir redécouvert l'Age $\mathrm{d}^{\prime} \mathrm{Or}^{21}$. L'échec suivit les premiers enthousiasmes et trois tentatives de colonisation de l'île de Roanoak, en 1585,1586 et 1587 , virent la cour se désintéresser de la colonie, malgré la présentation à la reine par Hakluyt de son Discourse on Western Planting, en octobre $1584^{22}$. Les arguments y plaidaient en faveur d'une affirmation des droits de la couronne, au nom des premières découvertes des frères Cabot, d'une extension du protestantisme contre 
l'expansion des puissances catholiques, des intérêts économiques de l'Angleterre. Il fallut attendre 1604 et la fin de la guerre avec l'Espagne pour que la charte royale accordée à la nouvelle "Virginia Company" encourageât les corporations londoniennes à participer aux expéditions virginiennes, sous le contrôle du "Royal Council for Virginia". Ce fut dans le cadre de ces tentatives colonisatrices qu'eut lieu en 1609 le naufrage du Sea Venture, navire-amiral d'une flotte de colons à destination de l'Amérique. Les naufragés, isolés neuf mois sur une certaine "Ile du Diable", finirent par atteindre Jamestown avant de revenir à Londres en 1610. Documents officiels et récits de voyage - les célèbres "Bermuda Pamphlets", connus de Shakespeare ${ }^{23}$ - donnèrent à l'aventure une dimension allégorique. L'allure diabolique de l'île des naufragés avait été démentie, de façon providentielle, par sa richesse paradisiaque et l'inspiration divine du gouverneur, qui avait su prévenir mutinerie, pillage et cannibalisme chez les naufragés. Une tempête intérieure, autrement plus dangereuse que la révolte des éléments, avait menacé la colonie de dissolution. Deux attitudes face à la colonisation devaient s'opposer à l'occasion de semblables mésaventures. Les partisans d'une plantation en Virginie avançaient des arguments d'ordre spirituel. Seuls méritaient le nom de coloni, colons ou cultivateurs de la glèbe, les planteurs soucieux de richesses spirituelles $^{24}$ - argument que devait reprendre Bacon ${ }^{25}$. Etaient dénoncés, par contre, aux côtés du Diable et des papistes, les opposants à l'aventure virginienne, dont les acteurs. Dans un sermon, prononcé en 1616 devant le gouverneur de Virginie, William Crashaw s'en prenait à ceux qui, selon lui, "injuriaient" le nouveau territoire :

But why are the Players enemies to this Plantation and do abuse it ? I will tell you the causes: First, for they are so multiplied here, that one cannot live by another, and they see that wee send of all trades to Virginea, but will send no Players, which if we would doe, they that remaine would gaine the more at home. Secondly, as the divell hates us, because wee purpose not to suffer Heathens and the Pope because we have vowed to tolerate no Papists; so doe the Players, because wee resolve to suffer no Idle Persons in Virginea, which course if it were taken in England, they know they might turne to new occupation. 26

Au rebours, les amis de Shakespeare conduisant la faction patriote au sein du "Virginia Council" - Henry Wriothesley, comte de 
Southampton, dédicataire de Venus and Adonis et de Lucrece, William Herbert, comte de Pembroke et Philip Herbert, comte de Montgomery, les dédicataires du premier in-folio du théâtre de Shakespeare - s'élevèrent contre les écueils et les dangers d'une aventure qui risquait de mettre en cause les fondements du royaume. Certains auteurs de pamphlets et dramaturges introduisirent dans leurs cuvres l'image d'une Virginie devenue symbole de virginité violée ${ }^{27}$.

Les spectateurs jacobéens de La Tempête étaient donc partagés entre le rêve de conquête coloniale et la fin d'un espoir d'appropriation de terres fertiles et de richesses minières, alors que, paradoxalement, l'Angleterre avait mis fin à la suprématie espagnole sur les mers. A entendre la description de son île par Caliban, où apparaissait en filigrane le parti-pris méditerranéen face à l'enjeu atlantique, ils découvraient la difficile alliance des civilisations de l'ancien et du nouveau monde. Dans l'œuvre de Shakespeare, où le nom de "Virginie" n'apparaît nulle part, la thématique de l'étranger ostracisé était intégré à une construction dramatique, dans le cadre d'une utopie.

La carte de Virginie, dessinée par Théodore de Bry, à partir de croquis de Walter Ralegh et de John White, explorateurs de 'lîle de Roanoak avec Thomas Hariot et autres savants, était une illustration graphique de la tentation d'établir une nouvelle Méditerranée en Amérique. Les noms indigènes de lieux voisinaient avec des noms latins et anglais. Le dessin introduisait, selon les méthodes cartographiques de l'époque, des images de chasse, de pêche, de culture ainsi que d'une cité-forteresse. La fascination pour les détails corporels et quotidiens traduisait le désir de mettre les terres nouvelles au service des découvreurs. L'île de Roanoak ne se regardait pas à partir d'un reflet inversé, comme l'île d'Utopie, mais à distance, à partir d'un promontoire imaginaire permettant d'introduire une demi-perspective, habituelle chez les cartographes élisabéthains ${ }^{28}$. En délimitant des pays nouveaux, dont l'existence était l'objet de suppositions depuis la fiction platonicienne de l'Atlantide, la géographie - le nom que le seizième siècle attribuait à l'art du cartographe - associait l'espace et le temps. Les navigateurs, cosmographes, savants et littérateurs retrouvaient un passé miraculeux et innocent, au travers des Caribéens. Les gravures en taille douce de Théodore de Bry, réalisées à partir des aquarelles de John White traitant des scènes quotidiennes en Virginie, mettaient 
face à face Indiens algonquins et Anglais. Quelques silhouettes de Pictes et de Bretons, dessinées à partir d'une remarque de William Strachey sur la ressemblance physique des ancêtres européens et des races nouvellement découvertes ${ }^{29}$, avaient l'allure de sauvages cannibales ${ }^{30}$. Dans ces planches, la représentation des Amérindiens mettait le spectateur face à ses propres interrogations quant aux conditions des plantations, chaque monde étant pour l'autre un "brave new world". Caliban, figuration éponyme des Caribéens, portait la marque de telles interrogations. Créature d'un monde préchristianisé, il correspondait aux descriptions d'un Pigafetta ou d'un Cortès. C'était un aborigène en voie de christianisation, dans la ligne de l'attitude pastoraliste, celle des Spenser, Sidney, Greville ou Drayton célébrant le renouveau de l'Age d'Or, grâce auquel les marchands avaient su retrouver la trace des apôtres. Dans le même temps, la créature shakespearienne conservait les traces du primitivisme, sous forme littéraire et iconographique. Il ne présentait aucune des caractéristiques physiques associées aux Amérindiens, puisqu'il ne recouvrait pas sa nudité d'une peau d'animal, ne portait ni plumes ni décorations fétiches et ne se livrait pas non plus au cannibalisme ${ }^{31}$. Par contre, il témoignait de la survivance d'une préoccupation humaniste quant à la "civility", aux conditions spirituelles de la confrontation entre les colonisateurs et les races nouvellement découvertes ${ }^{32}$. Caliban s'apparentait à ces créatures bestiales qui hantent les forêts selon Spenser, ces "wild men" sur qui aucune emprise spirituelle n'est jamais possible - «a satyre sonne yborne in forrest wyld.» (The Fairie Queene, I. vi. 21.1). Prospero dénonçait en lui le retour, toujours possible, de la bestialité :

A devil, a born devil, on whose nature

Nurture can never stick; on whom my pains,

Humanely taken, all, all lost, quite lost ;

And as with age his body uglier grows,

So his mind cankers.

(IV. i. 188-92)

L'homme sauvage dont la nature s'oppose à tout effort civilisateur, s'éloignait ainsi à tout jamais du "foundling", la créature nouvellement redécouverte, image de l'homme ancien sur qui fonder la renaissance de l'homme nouveau. Paradoxalement, pourtant, Caliban était aussi la représentation de l'être fruste, sauvage, en espoir de salut, digne d'un effort de sauvetage spirituel, où la sauvegarde ("salvage") est une étape vers le salut ("salvation"). Quel que fût le 
degré d'échec de Prospero face à son esclave indigne, Caliban entrait dans la problématique des découvertes.

Par ailleurs, les parallèles que l'on peut établir entre l'histoire politique de l'Angleterre aux seizième et dix-septième siècles et certains éléments de l'intrigue de La Tempête ne manquent pas. Ainsi, la scène où Ferdinand et Miranda apparaissent en train de jouer aux échecs propose-t-elle une illustration dramatique de la description, par Thomas More, des jeux, proches du jeu d'échecs, pratiqués par les Utopiens, en particulier «le jeu de conflit, par bataille rangée, des vertus et des vices» $(U t ., \mathrm{p} 470)^{33}$. Comme la scène maintient, chez le partenaire masculin, une part de tricherie, elle semble ainsi reprendre la partie d'échecs interrompue, près d'un demi-siècle auparavant, par Henri VIII, lorsqu'il fut informé de la décapitation, sur son ordre, de son conseiller et ami Thomas More. On y retrouve aussi en filigrane, une autre partie d'échecs politique, à savoir celle qui fut entreprise par Sir Walter Ralegh, lorsqu'il fut placé en liberté conditionnelle par Jacques $1^{\text {er }}$ en 1617 pour aller tenter, au péril de sa vie, une nouvelle expédition en Amérique. Son échec devait lui être fatal, puisqu'il fut décapité à son retour. Cela se passait un siècle après que Bartholomé de Las Casas eut présenté à Charles Quint un de ses projets civilisateurs, en grande partie influencé par la lecture de l'Utopie de Thomas More. La tentative de créer une nouvelle Jérusalem, libérée de toute violence, à la différence des territoires espagnols - fut décrite par Walter Ralegh comme un "douloureux pèlerinage"34. L'entreprise imaginaire de Prospero, à l'image de celle, réelle, de l'auteur de The Discoverie of Guiana, sous l'égide de Jacques $1^{\text {er }}$, se heurte elle aussi à de sombres conjurations politiques.

En dernière analyse, La Tempête apporte un commentaire allégorique sur le topos de la conquête des îles, une constante des récits de voyage depuis Christophe Colomb. Le navire royal que l'art de Prospero fait disparaître devant l'île magique tient à la fois des vaisseaux fictifs partis vers l'île d'Utopie et des vaisseaux véritables que la plume de Théodore de Bry représente, et dont cinq sombrèrent devant l'île de Roanoak. A partir d'un naufrage symbolique, l'intrigue shakespearienne dénonce implicitement les dystopies qui détruisent les royaumes. Le jeu de mots possible sur "king'ship" et "kingship" foumit à lui seul un commentaire sur les enjeux de La Tempête. La séparation physique du "roi" et de son "navire", essentielle à la mise en scène de Prospero, traduit méta- 
phoriquement la coupure nécessaire entre l'usurpateur et l'emblème du pouvoir usurpé (I. ii. 196, 224, 227, 236). Lors du dénouement, la victoire du duc de Milan sur ses adversaires se traduit par la réapparition du navire sans ses passagers indignes. La métaphore nautique révèle alors un Prospero à nouveau maître de son duché (V. i. 97, 222, 237, 307) ${ }^{35}$. La thématique centrale de La Tempête ne serait-elle pas centrée sur l'idée d'un divorce inévitable entre le souverain et son royaume, dès lors que la justice en a quitté les rives? L'île de Prospero, comme l'île d'Angleterre, est un lieu d'ambiguittés, de contradictions, de paradoxes. Vue de l'extérieur sa représentation allégorique est une série d'écueils qui, comme la montagne initiatique, représente les tentations qui assaillent l'âme. Sur le plan anagogique, l'île-refuge s'ouvre sur l'intérieur. La cellule de Prospero, île à l'intérieur de l'île, à la façon des îles creuses de Thévet ${ }^{36}$, assure une communication avec les abysses d'un passé disparu. Tempête, mer, rivages, eau et limon sont alors emblématiques d'un voyage de l'Esprit. Le voyage de Prospero qui, selon le vœu de Gonzago, devrait être inscrit en lettres d'or sur des colonnes à l'épreuve du temps, est en fait une quête de Connaissance :

In a poor isle, and all of us ourselves

When no man was his own.

(V. i. 212-3).

La conquête de l'île, dans l'ancien comme dans le nouveau monde, relève de la prudence d'humaniste et doit être envisagée, à la façon de lirréductible et vertueux courtisan, conseiller du roi. Le souvenir de l'auteur de l'Utopie ne hante-t-il pas l'auteur de $L a$ Tempête?

Raymond GARDETTE

Université de Paris-Sorbonne

\section{NOTES}

1. Le meilleur exemple est le Navigatio Sancti Brendani, texte mystique du dixième siècle, qui décrit une expédition monastique irlandaise vers la terre promise. La découverte d'îles au climat adouci et riches de merveilles naturelles - Terre Neuve, les Açores, les Bahamas - propose une version idéalisée de l'Irlande. Voir John D. Anderson, "The Voyage of Brendan, an Irish Monastic expedition to Discover the 
Wonders of God's World", The American Benedictine Review, 43 (1992), 262-82.

2. Les références traditionnelles à Ophir (I - Rois : IX, 28 ; I Chroniques: XXIX, 4 ; II - Chroniques: VIII, 18 \& IX, 10 ; Psaumes : XLV, 21 ; Livre de Job : XXVIII, 16 ; Livre d'Isaïe : XIII, 12) entrent dans la description anagogique du royaume céleste. L'or de Salomon signifie l'aide divine aux vrais croyants. Voir : Brazen de la Martinière, Le Grand dictionnaire géographique et critique, 10 vol. (La Haye, 1726-1739), s.v. "Ophir".

3. Hakluytus Posthumus, or Purchas His Pilgrimes, contayning a History of the World in Sea Voyages and lande Travelles by Englishmen and Others (1625), "Q1 : The Allegorical and Anagogicall sense or application of Solomon Ophirian Navigation", Sig. 132. La page de garde de cette version élargie de la première compilation Purchas His Pilgrimage, or Relations of the World and the Religions observed in all Ages and Places discovered from the Creation unto this Present (1613, 2nd ed. 1614) - représente Jacques $1^{\text {er }}$ et le prince Charles, symétriques de la reine Elisabeth et du prince Henri, autour d'une image allégorique d'Israël en marche vers la Nouvelle Jérusalem. Trente médaillons de voyageurs célèbres, de Nabuchodonosor à Colomb et aux navigateurs contemporains surmontent les deux hémisphères et un portrait de l'auteur.

4. Les citations de La Tempête sont empruntées à l'Edition de Stephen Orgel (The Oxford Shakespeare, Oxford University Press, 1987).

5. Thomas More, De optimo reipublicae statu, deque nova insula Utopia (De la République en son meilleur état, et de l'île nouvelle d'Utopie) Londres, 1516. Trad. française par Jean Leblond: La description de l'isle d'Utopie où est le miroër des républiques du monde (Paris 1550); (rééd. E. Jeanneret, Mouton, la Haye, 1970 \& M.A. Screech, Mouton, Londres, Johnson Reprint Corporation, "Classiques de la Renaissance en France", 1970. Traductions modemes de référence, Marie Delcourt, Utopia (Genève, Droz, 1936) et André Prévost, l'Utopie de Thomas More (Paris, Nouvelles Editions Mame, 1978) - notre édition de référence, ci-après : $U t$.

6. Sur les rapports entre équité et justice, voir B. Tannier, "La justice dans The Fairie Queene de Spenser" in Justice, Juges, Prisons dans le théâtre de Shakespeare et dans les cuures de ses contemporains. ed. M.-T. Jones-Davies. (Société Française Shakespeare, Actes du Congrès 1980, Paris, J. Touzot, 1981), pp. 9-21.

7. Voir l'introduction à The Tempest, ed. par Stephen Orgel, The Oxford Shakespeare (Oxford: O.U.P., 1987), 1-87. Du même auteur: "Prospero's wife", in S. Greenblatt, ed. Representing the English Renaissance (Berkeley, Los Angeles et Londres, Univ. of California Press, 1988), pp. 217-29. 
8. Voir Louis Marin, Des Pouvoirs de l'Image. Gloses (Paris, Seuil, 1993), pp. 169-85 : "Entreglose 4. Le portrait du poète en roi".

9. Ut., 34 et Arthur W. Reed, "John Rastell's Voyage in the year 1517", The Mariner's Mirror, 9 (1923), 137-8.

10. Thomas More emprunte des détails physiques à Waldseemüller, Cosmographiae Introductio et aux Quatuor Americi Vespucij navigationes (Les Quatre navigations d'Amerigo Vespucci), imprimés conjointement par Walter Ludel le 25 avril 1507. Traduction moderne : La Fortune d'un nom AMERICA ... Cosmographiae Introductio suivi des lettres d'Amerigo Vespucci, texte présenté par Albert Ronsin (Grenoble, Jérôme Millon, 1991), p. 185: «Chez les écrivains anciens on ne parle pas d'elles, je crois, parce qu'ils n'en eurent pas connaissance ; parce que, si je m'en souviens bien, dans l'un d'eux j'ai lu qu'il était d'avis que cette mer Océane était sans habitants. De cette opinion fut Dante notre poète, dans le $\mathrm{XXVI}^{\mathrm{e}}$ chapitre de son Enfer où se trouve la fiction de la mort d'Ulysse».

11. Christoph Strosetzki, 'L'Utopie de Thomas More: une réponse au débat sur le Nouveau Monde", Moreana, XXVII, 101-102 (Mai 1990), 5-24.

12. Stephen Orgel, "Prospero's Wife" Representations 8 (1984) 1-13 ; repr. in Representing the English Renaissance, ed. S. Greenblatt (Berkeley, Los Angeles and London. Univ. of California Press, 1988), pp. 217-19, "A Bibliographical Coda" (pp. 228-9). Par une argumentation convaincante, l'auteur propose la lecture "wife" plutôt que "wise".

13. François Laroque, "En marge de l'idéologie: antimasque et grotesque dans le Dr Faustus et La Tempête", in Théâtre et Idéologie : Marlowe, Shakespeare, ed. M.T. Jones-Davies (Société Française Shakespeare, Paris, J. Touzot, 1982), pp. 99-114.

14. Mireille Pastoureau, Voies océanes. Carte marines et grandes découvertes (Paris, Bibliothèque Nationale, 1992) p. 14.

15. Walter W. Skeat, A Concise Etymological Dictionary of The English Language (Oxford, Clarendon Press, 1911), s.v. "Island".

16. Jacques Gury, "About the maps of Utopia", Moreana, $n^{\circ} 42$ (Juin 1974), 99-101. Alain Bony, "'Fabula, Tabula': L'Utopie de More et l'image du monde", Etudes Anglaises, XXX, n 1 (Janv. Mars 1977), 1-19. L'auteur reproduit face à face les cartes d'Utopie, de 1516, par Gérard Geldenhauer et celle d'Ambrosius Holbein, pour l'édition de 1518. Reproduites également in Prévost, Ut., pp. 221 et 332 et in E. Surtz and J. H. Hexter, eds., The Complete Works of St Thomas More, vol. 4, Utopia (New Haven, London, and Yale Univ. Press, 1965), pp. 16-17.

17. Voir Louis Marin, Utopiques. Jeux d'espace. (Paris, Ed. de Minuit, 1973). 
18. Les Essais de Montaigne, éd. Villey-Saulnier (Paris, P.U.F., 3e éd., 1978), livre I, Chapitre XXXI, "Des Cannibales", p. 206.

19. Citation in "Des Cannibales", p. 213 : «lls dirent qu'ils trouvaient en premier lieu fort estrange que tant de grands hommes, portans barbe, forts et armez, qui estoient autour du Roy (il est vraysemblable que ilsl parloient des Suisses de sa garde), se soubsmissent à obeyr à un enfant, et qu'on ne choisissoit plus tost quelqu'un d'entr'eux pour commander; secondement (ils ont une façon de leur langage telle, qu'ils nomment les hommes moitié les uns des autres) qu'ils avoyent aperçeu qu'il y avoit parmy nous des hommes pleins et gorgez de toutes sortes de commoditez, et que leurs moitiez estoient mendiants à leurs portes, décharnez de faim et de pauvreté ; et trouvoient estrange comme ces moitiez icy necesssiteuses pouvoient souffrir une telle injustice, qu'il ne prinsent les autres à la gorge, ou missent le feu à leurs maisons" (Ibid., pp. 213-4).

20. R. Gardette, "Ariel et Caliban: L'imaginaire monstrueux dans La Tempête de William Shakespeare" in Monstres et Prodiges au temps de la Renaissance, ed. M.-T. Jones-Davies, Centre de Recherches de la Renaissance, (Paris, Jean Touzot, 1980), pp. 69-80.

21. David Beers Quinn, The Roanoke Voyages, 1584-1550: Documents to illustrate the English Voyages to North America Under the Patent Granted to Walter Raleigh in 1584 (Cambridge, Hakluyt Society, 2nd ser., nos 104-5, 1955; photolithographic reprint in one vol., Nendeln, Liechtenstein, 1967), pp. 91-126. (Rapport d'Arthur Barlow). Egalement in Hakluyt's Voyages. A Selection by Richard David (London, Chatto \& Windus, 1981), pp. 445-53 : The first voyage made to the coasts of America with two barks, wherein were captains Master Philip Amadas, and Master Arthur Barlow, who discovered part of the country now called Virginia, anno $1584 \ldots$ :

p. 117 : «he country, Wingandacoa, and now, by her Majesty, Virginia»;

p. 450 : «We found the people most gentle, loving and faithful, void of all guile and treason, and such as live after the manner of the golden age».

22. D. B. Quinn, England and the Discovery of America, 14811620 (London, G. Allen \& Unwin, 1974), pp. 293-4.

23. Extraits in F. Kermode, ed., The Tempest, (New Arden Shakespeare), pp. 135-41; S. Orgel, ed., The Tempest (The Oxford Shakespeare), pp. 209-19 \& Geoffrey Bullough, Narrative and Dramatic Sources of Shakespeare (London, Routledge \& Kegan Paul, N.Y., Columbia Univ. Press, vol. VIII, 1975), pp. 275-98.

24. J. P. Brockbank, The Tempest : Convention of Art and Empire, "The island of The Tempest" in J. P. Brockbank, On Shakespeare (Oxford, Basil Blackwell, 1989), pp. 303-21, 322-40. 
25. F. Bacon, Essays : XXX iii, "Of Plantations": «I like a plantation in a pure soil ; that is, where people are not displanted, to the end to plant in others; for else it is rather an extirpation than a plantation.» Bacon appuie sa démonstration sur l'exemple de la Virginie. "Pure soil" fait indirectement référence à la lettre patente d'Elisabeth à Ralegh, du 25 mars 1584: «We do give and grant to Walter Ralegh Esquire and to his heirs and assigns for ever, free liberty to search, find out, and view such remote heathen and barbarous lands, not actually possessed of any Christian Prince nor inhabited by Christian people, as to him as shall seem good: and the same to have hold ocupy and enjoy...» (Lettre reproduite in Sir Robert H. Schomburgk, ed., The Discoverie of the Large, Rich and Bewtiful Empire Of Guiana, with a Relation of the Great and Golden City of Manoa (which the Spaniards call El Dorado) ... Performed in the yeare 1595. by Sir W. Ralegh, Knight. (London, The Hakluyt Society, First series, III, 1848 ; reprint, New York, Lenox Hill, 1970).

26. William Crashaw, A Sermon preached in London before the right honourable Lord Lawarre, Lord Governor and captaine generall of Virginia (Title-page : A New-yeeres gift to Virginea), 1610, Sig $\mathrm{H}^{4}$.

27. Des allusions, chez Spenser, Sidney, Dekker, Nashe mettent en cause les motivations commerciales des premiers essais de colonisation. Un certain nombre de situations parodiques, dans le théâtre de Marston, Jonson, Middleton ou Massinger décrivent les attitudes mercantiles déguisées en souci de découverte de terres vierges. Voir, par exemple Jonson, Chapman, Marston, Eastward Ho!, New Mermaid, III; iii. 14-16:

«Seagull : Come, boys, Virginia longs till we share the rest of her maidenhead.

Spendall : Why, is she inhabited already with any English?»

28. D. B. Quinn, The Roanoak Voyages, op. cit.; P. Hutton and Quinn, D. B., The American Drawings of John White, 1577-1540, 2 vols (London, The Trustees of the British Museum \& Chapell Hill, Univ. of North Carolina Press, 1944); M. Bouyer et J. P. Duviols, Le Théâtre du nouveau monde. Les grands voyages de Théodore de Bry (Paris, Gallimard, "Découvertes Gallimard Albums", 1992), pp. 6 et 136-7, planche II. La carte, gravée à partir d'un dessin disparu de John White, représente le littoral, du cap Hatteras à l'île de Knott.

29. The historie of travell into Virginia Britania, by William Strachey, Gent (1612), eds. L. B. Wright and V. Freund (London, Hakluyt Society Publications, 2nd series 103, 1953). Strachey propose quelque huit-cents mots de la langue algonquienne.

30. Le théâtre du nouveau monde p. $37-47$, planches I. V : "Quelques portraits des Pictes, anciens habitants d'une partie de la Bretagne." 
31. Voir, par exemple, C. Frey, "The Tempest and the New World", Shakespeare Quarterly, 30 (1979), 19-41 ; A. T. Vaughan, "Shakespeare's Indian: The Americanization of Caliban", Shakespeare Quarterly, 39 (1988), 137-53; M. A. Skura, "Discourse and the Individual : The case of Colonialism in The Tempest", Shakespeare Quarterly, 40 (1989), 42-69.

32. A. Bartlett Giamatti, "Primitivism and the Process of Civility in Spenser's Fairie Queene" in F. Chiappelli, ed. First Images of America, 2 vols (Berkeley, Los Angeles, London, University of California Press, 1976), vol. 1, pp. 71-82.

33. Sur les rapports entre l'Utopie et La Tempête, voir : $M$. Holquist, "How to Play Utopia: Some Brief Notes on the Distinctiveness of Utopian Fiction", Yale French Studies, 41 (1968), 10623 ; H. Felperin, Shakespearean Romance (Princeton, NJ, 1972), pp. $246-83$; K. Flagstad, "Making this Place Paradise : Prospero and the Problem of Caliban", Shakespeare Studies, XVIII (1986),205-33.

34. The Discoverie ... of Guiana, ed. Sir Robert M. Schomburgk (Hakluyt Society, First Series, 3, 1848). Op. cit. : Epistle Dedicatory, p. V. Ralegh décrit son expédition comme un "painful pilgrimage". Dans le récit, Manoa, ou El Dorado, apparaît comme une Jérusalem céleste. Voir: Mary B. Campbell, The Witness and The Other World. Exotic Travel Writing, 400-1600 (Ithaca and London, Cornell Univ. Press, 1988), pp. 211-54 : "6-Inward Feeling": Ralegh and the Penetration of the Interior".

35. The Oxford English Dictionary,-s.v. "-ship suffix". Par étymologie, le suffixe traduit l'état, la condition, le rang, la qualité de la personne, voire son occupation. Dans cette demière acception, le premier usage de "courtship" est shakespearien. Le jeu de mots à partir de la polysémie de "ship", à la fois substantif et suffixe, est habituel chez Shakespeare. Voir, par exemple, "mastership" et "master's ship", dans The Two Gentlemen of Verona (New Arden Shakespeare), III. i. 279-80 et le néologisme créé par Iago "his Moorship's Ancient", Othello (New Arden Shakespeare), I. i. 33 (texte de l'in-folio, non retenu dans l'édition Arden).

36. Voir: Frank Lestringant, "Les Iles creuses de l'archipel (l'Insulaire d'André Thévet)" in François Moureau, ed. L'île territoire mythique (Paris, Aux Amateurs de Livres, 1989), pp. 19-26. 